\title{
Techniques, technologies, apprentissage et plaisir au travail...
}

Techniques, Technologies, Training and Pleasure in Work ...

\section{François Sigaut}

\section{(2) OpenEdition \\ 1 Journals}

Édition électronique

URL : https://journals.openedition.org/tc/4770

DOI : $10.4000 /$ tc. 4770

ISSN : 1952-420X

Éditeur

Éditions de l'EHESS

\section{Édition imprimée}

Date de publication : 1 décembre 2009

Pagination : 40-49

ISBN : 978-2-7351-1301-9

ISSN : 0248-6016

\section{Référence électronique}

François Sigaut, «Techniques, technologies, apprentissage et plaisir au travail... », Techniques \& Culture [En ligne], 52-53 | 2009, document 1, mis en ligne le 01 août 2012, consulté le 29 septembre 2022.

URL : http://journals.openedition.org/tc/4770 ; DOI : https://doi.org/10.4000/tc.4770 


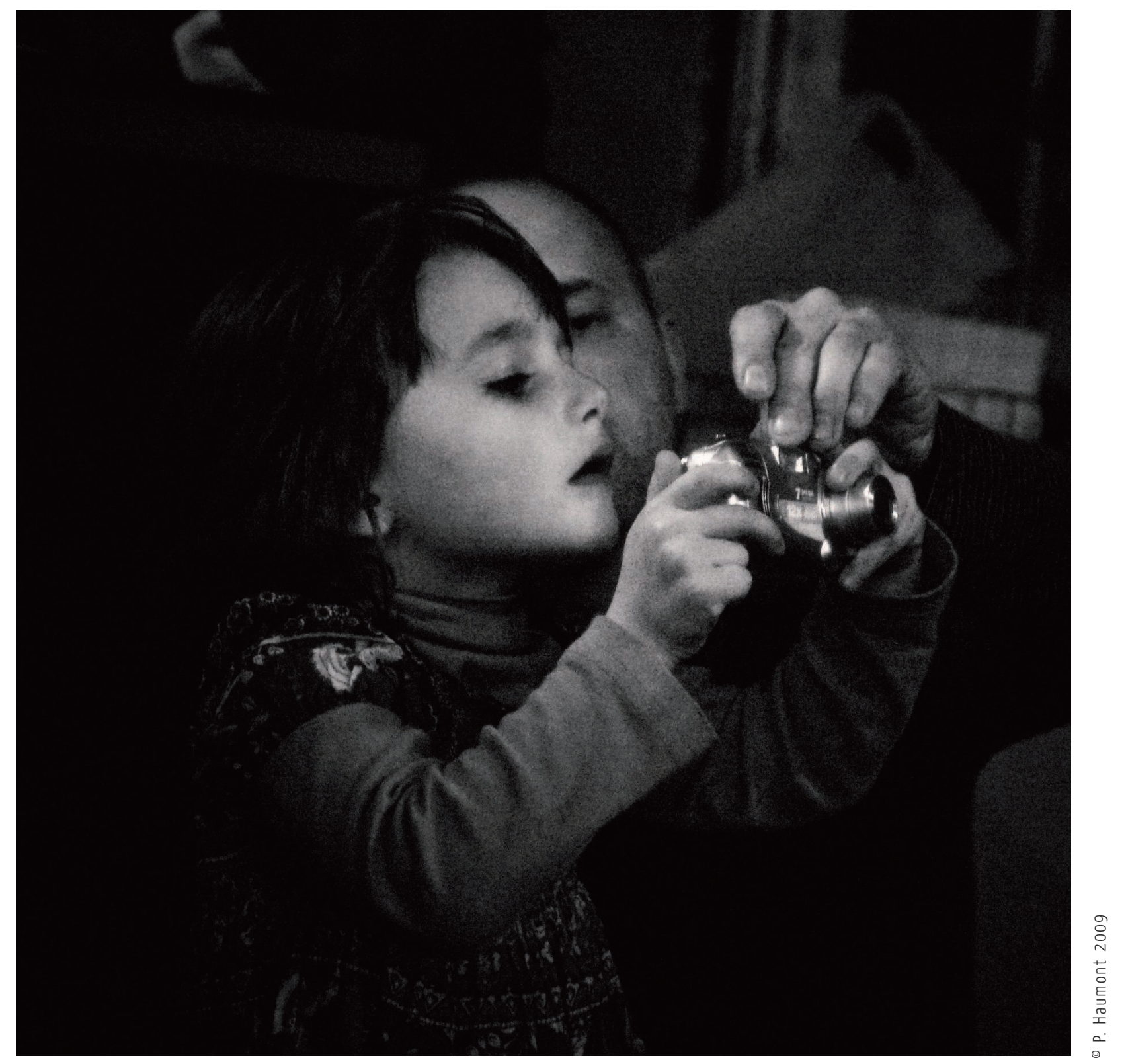




\section{TECHNIQUES, TECHNOLOGIES, APPRENTISSAGE ET PLAISIR AU TRAVAIL...}

Dans l'espèce humaine, le lien social le plus fondamental est celui qui naît de l'expérience partagée. Une expérience qui est, qui ne peut être que celle de l'efficacité matérielle. Car il faut qu'une action soit matériellement efficace, c'est-à-dire qu'elle produise un résultat visible, tangible, perceptible par tous, pour qu'elle ait valeur d'expérience.

La recherche en anthropologie des techniques - au sens large, incluant l'ethnologie, l'histoire, la géographie, la sociologie, la linguistique, etc., c'est-à-dire toutes les sciences sociales - présente d'innombrables difficultés, bien connues de ceux qui se sont engagés dans cette voie peu fréquentée. Dans cet article, je voudrais parler de quelques-unes de ces difficultés, qui, à tort ou à raison, me paraissent sous-estimées ou négligées. Ces difficultés viennent pour la plupart des idées souvent partielles que les uns et les autres se font de la technique.

Première difficulté: la confusion entre technique et technologie. Le problème ne se pose pas tout à fait dans les mêmes termes en anglais, en allemand, en français et dans les autres langues, qui ont chacune, sur ce point, leurs traditions propres. Ces traditions ne peuvent ni ne doivent être ignorées, il est au contraire indispensable de les analyser avec soin. Mais ces analyses ne nous donneront pas la solution du problème tel qu'il se pose à nous chercheurs. Toutes les disciplines scientifiques ont besoin 
*Sur l'apprentissage Les recherches sur l'apprentissage se sont multipliées à partir du milieu des années 1980. La littérature sur le sujet est abondante : l'ouvrage cité plus haut, Savoir-faire et pouvoir transmettre (1991) comporte une bibliographie cumulée de 472 titres, à laquelle je renvoie le lecteur. Tout ce que je peux faire ici est de citer les noms de quelques auteurs qui y figurent et qui me paraissent particulièrement marquants (par ordre chronologique): Chamoux 1978, Salmona 1983, Delbos \& Jorion 1984, Giordan \& de Vecchi 1987, Bureau \& de Saivre 1988, Rogoff 1990, Lave \& Wenger 1991, liste à laquelle il faudrait ajouter des ouvrages parus ultérieurement, comme par exemple Guédez, Annie, 1994, Compagnonnage et apprentissage, Paris, PUF. de se doter d'un vocabulaire précis et nettement défini (aussi nettement que possible), et toutes doivent donc, à un moment ou à un autre, arriver à décider du choix de leurs mots et du sens qu'elles leur donnent. Nous qui nous intéressons aux techniques ne faisons pas exception. L'analyse des diverses traditions de langage ne nous dispense pas du devoir de décider pour notre propre compte. En France, nous sommes quelques-uns, depuis Mauss, Haudricourt, et Leroi-Gourhan, à adhérer à la décision suivante: nous appelons technique l'« action traditionnelle efficace », et nous appelons technologie la science (sociale) qui prend la technique pour objet. Cette solution a le mérite de la clarté et de la simplicité. Il se peut qu'elle soit plus difficile à faire passer dans les pays de langue anglaise qu'en France (encore que...), et mon propos n'est certes pas de dire à nos collègues anglophones ce qu'ils doivent faire. Ils sont libres, chacun est libre de prendre la décision qui lui paraît la meilleure, à la seule condition de l'expliciter et de la justifier. La seule liberté qui nous soit interdite, c'est celle de ne rien décider, parce que cela reviendrait à accepter une confusion incompatible avec ce qu'il y a de plus fondamental dans la démarche scientifique.

Il existe d'ailleurs un autre mot qui complète les deux précédents, et dont l'utilité me semble peu contestable: c'est celui de technographie. Ce mot fut employé dans les années 1880 et 1890 par des auteurs comme Franz Reuleaux en Allemagne et Otis Tufton Mason aux États-Unis. Avant Reuleaux et Mason, le terme technographie avait été proposé dès 1838 par André-Marie Ampère dans son essai sur la philosophie des sciences (je dois cette information à Joost Mertens). Mais ce fut apparemment sans succès. Technographie est à technologie à peu près ce qu'ethnographie est à ethnologie. On peut regretter que ce mot ne soit pas entré dans l'usage courant. En revanche, cela a eu l'avantage de ne pas donner lieu aux mêmes confusions : technographie fait référence à la tâche qui consiste à décrire les techniques, et à rien d'autre. Tâche essentielle et suffisamment difficile pour que plusieurs auteurs (dont Reuleaux lui-même et à nouveau Leroi-Gourhan $\&$ al.) aient consacré beaucoup d'efforts à en préciser les conditions et les méthodes. Ce thème est si important et si complexe qu'un colloque, voire plusieurs, ne seraient pas de trop pour arriver à y voir clair. En attendant, qu'on me permette la brève remarque suivante.

\section{L'Apprentissage"}

Une technique n'est vraiment connue que quand on en a acquis l'expérience, par un apprentissage plus ou moins long. Or personne ne peut multiplier les apprentissages au-delà d'un nombre qui restera toujours très limité par rapport à l'ensemble des apprentissages possibles. Dès lors, comment parvenir à une connaissance d'ordre véritablement général des techniques, ce qui est le but spécifique de la technologie? La technologie n'est-elle pas tout simplement impossible? Ainsi posée, la 
question est évidemment décourageante (comme toutes les questions trop générales). Je crois cependant que le problème n'est pas insoluble, à condition qu'on accepte de le poser, ou plus exactement de l'expliciter. Tous les chercheurs se sont heurtés un jour ou l'autre à la difficulté de communiquer leur expérience par les moyens ordinaires de l'écrit, de l'oral et même de l'image, et tous aussi ont pu se trouver dans l'embarras de s'apercevoir, au moment de la communiquer, que leur expérience était incomplète ou insuffisante. C'est que l'apprentissage du technologue (ou du technographe) se situe à deux niveaux: il doit apprendre à faire, et en plus, il doit apprendre à expliquer à d'autres comment on fait - à d'autres qui n'ont pas et ne pourront pas avoir la même expérience que lui.

Notre problème serait réellement insoluble si ces autres n'avaient aucune expérience technique d'aucune sorte. Je ne crois pas que cela puisse être. Peut-être cela sera-t-il un jour, quand nous serons environnés de machines tellement empressées à satisfaire tous nos besoins qu'il ne nous restera plus rien d'autre à faire que d'appuyer sur un bouton de temps à autre - à moins que l'implantation de puces dans nos organes ne permette de supprimer cette dernière formalité. Mais nous n'y sommes pas encore. Ce qu'on peut appeler la culture technique ordinaire, celle que chacun acquiert dans ses activités quotidiennes, reste un élément fondamental de la condition humaine. Que cette culture ordinaire soit souvent très insuffisante, et cela d'autant plus que l'enseignement scolaire et les médias font tout pour la réduire à l'insignifiance, c'est un autre débat. Mais elle existe, et tant qu'elle n'aura pas disparu, la communication de l'expérience technique sera un problème sans doute difficile, mais pas insoluble. Un des objectifs de la technographie est en tous cas la recherche des solutions à ce problème. Je viens de faire allusion à l'expérience technique et à son acquisition par l'apprentissage. Il s'agit là de deux thèmes d'une importance tout à fait primordiale pour la technologie. Je leur ai consacré plusieurs articles, notamment dans Techniques $E$ culture, aussi vais-je me permettre de résumer mon propos à l'extrême. Le savoir-faire ne s'acquiert que par expérience personnelle. On ne peut pas se contenter de regarder faire autrui, même si ce regard joue un rôle essentiel. Il faut passer à l'acte. Or on n'expérimente pas seul (ce qui est le propre du savant fou dans nos romans de science-fiction). L'expérience est aidée, guidée, facilitée par ceux qui l'ont déjà acquise, et c'est cette aide, ce guidage qui sont socialement institués dans l'apprentissage. Dans l'apprentissage, le groupe transmet à ses nouveaux membres la somme des expériences que ses membres partagent entre eux et qui les identifient comme tels. Et de ce fait, l'apprentissage n'est pas seulement acquisition de savoirs, c'est aussi l'acquisition d'une identité, celle de membre du groupe où ces savoirs sont reconnus et valorisés parce que partagés.

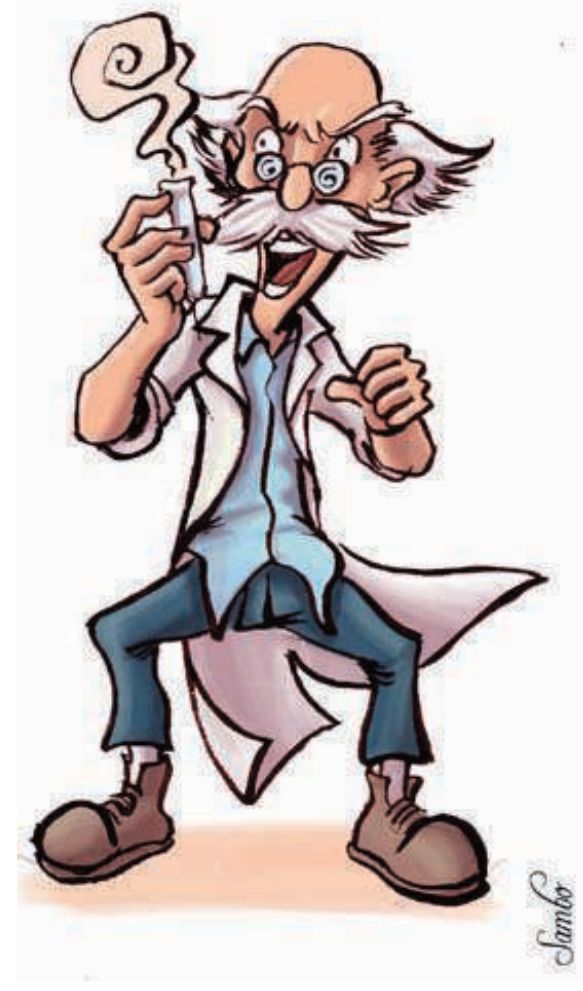


La vis secrète des jeunes

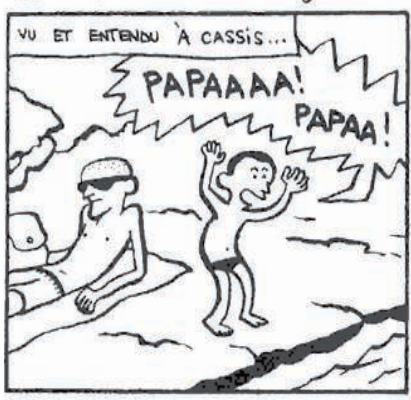

* Sur l'expérience partagée Ici en revanche, la bibliographie est particulièrement pauvre. En dehors des articles que j’ai moi-même consacrés à ce sujet (Sigaut 1990-2004, 1992 et 2007), le seul ouvrage qui en traite explicitement (à ma connaissance) est celui de Jean-Pierre Darré (cf. bibliographie).
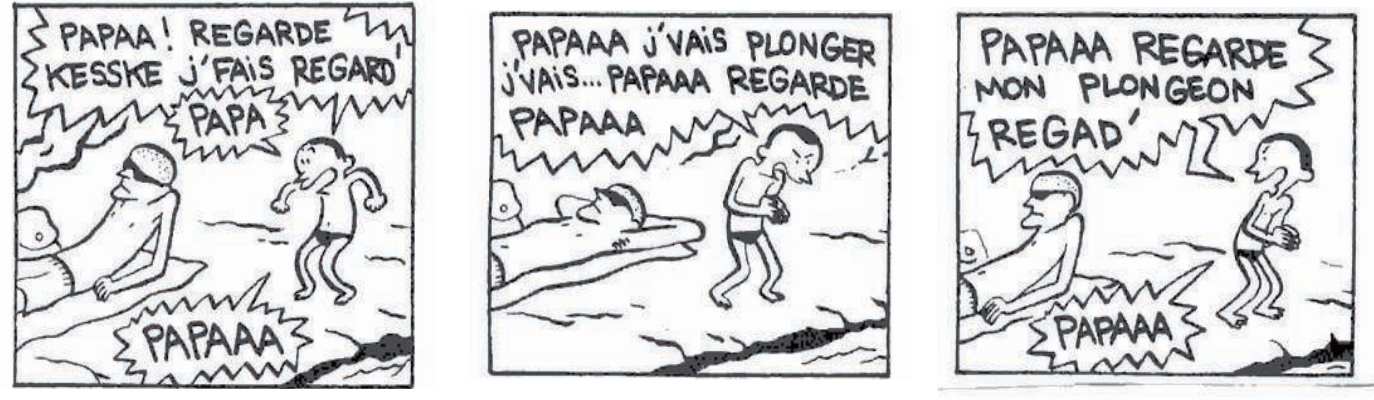

\section{L'expérience partagée"}

Cette notion d'expérience partagée est, je crois, fondamentale. C'est elle qui est à la base du sens commun du ou des groupes sociaux auxquels j'appartiens. Le sens commun (ce qu'on appelle culture n'en est qu'une manifestation) est ce qui fait la cohésion du groupe et son identité. C'est la grammaire des façons de faire à laquelle je dois me conformer plus ou moins. Si je m'en écarte, on me corrigera d'une façon ou d'une autre. Mais si je persiste, je risque d'être exclu, exclusion qui pourra aller jusqu'à l'imputation de folie. Il se peut que je sois vraiment fou et on parle alors, à juste titre, d'aliénation mentale. Mais il se peut aussi que je sois traité comme fou parce que mon expérience, quoique réelle, est incompréhensible ou inacceptable par les autres: j'ai proposé de parler dans ce cas d'aliénation sociale (celle, encore une fois, qui caractérise le savant fou, le " mad scientist »). Il y a enfin un troisième cas, dans lequel c'est le groupe lui-même qui refuse de prendre en considération certaines réalités parce qu'elles pourraient déranger son fonctionnement interne, et j'ai proposé pour cela le terme d'aliénation culturelle. J'ai bien conscience des difficultés d'une présentation aussi schématique. Ce que je voudrais qu'on en retienne, c'est que dans l'espèce humaine, le lien social le plus fondamental est celui qui naît de l'expérience partagée. Une expérience qui est, qui ne peut être que celle de l'efficacité matérielle. Car il faut qu'une action soit matériellement efficace, c'est-à-dire qu'elle produise un résultat visible, tangible, perceptible par tous, pour qu'elle ait valeur d'expérience. Et c'est à cette condition que l'expérience peut devenir tradition.

Mais efficace ne veut pas dire utile, et il y a là une autre confusion dont il faut se garder. L'utilité est une notion dont il ne faut assurément pas sousestimer l'importance, mais qu'il ne faut pas considérer comme si c'était une valeur en soi, indépendamment des autres. Laction technique est toujours efficace, en ce sens que pratiquée avec compétence et dans des conditions normales, elle produit le résultat ou l'effet prévu; si cela n'était pas le cas, elle n'aurait aucun sens. Mais l'action technique n'est pas toujours utile, au sens économique du terme. On peut la pratiquer dans le but de s'exercer, 

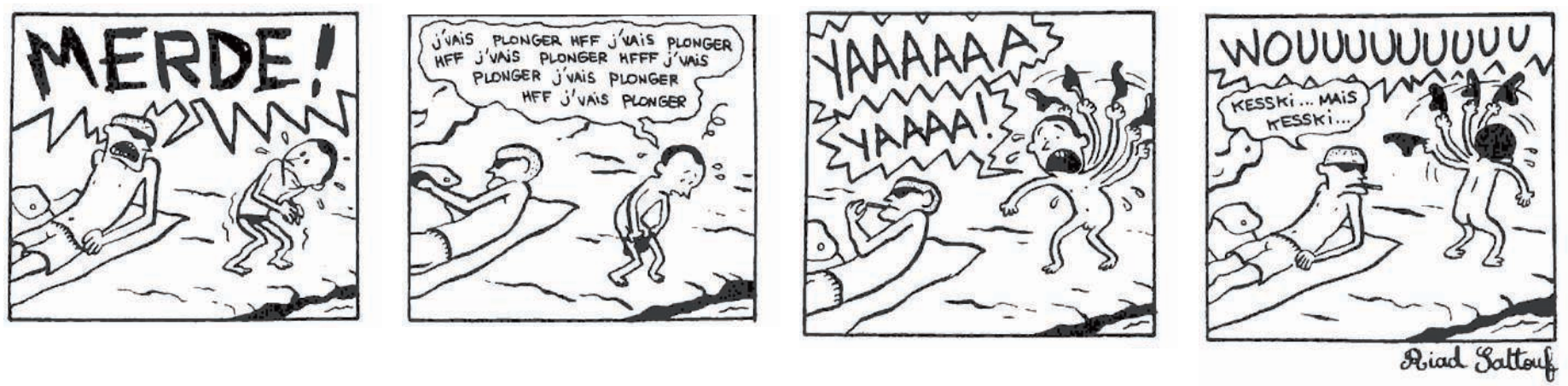

Charlie Hebdo $\mathrm{n}^{\circ} 278$ du 30 mai 2006

et l'effet est alors apprécié pour lui-même, pour sa valeur de réussite, parce qu'il témoigne de l'habileté du sujet. L'exercice est une forme d'apprentissage pour les actions qui sont à la fois difficiles et non répétitives, comme la chasse ou la guerre. On peut dire que l'utilité y est seulement différée: je m'exerce (au tir) pour que mon action soit utile le moment venu (à la chasse ou à la guerre). Mais de l'exercice au jeu, il n'y a qu'un pas, et ce qui distingue le jeu proprement dit, c' est qu'il n'y a plus d'utilité du tout, même différée: le plaisir de la réussite est l'unique finalité de l'action. C'est ce qu'on peut constater tous les jours au spectacle des sports modernes. Que la plupart des sports mobilisent aujourd'hui des flux financiers considérables, c'est évident mais cela n'infirme nullement ce que j'essaie de montrer. Améliorer d'un dixième de seconde le record du 100 mètres ne sert évidemment à rien, si ce n'est à montrer que quelqu'un en a été capable. Qu'il s'agisse de tennis, de football, de natation ou de patinage artistique, les sportifs jouent pour leur plaisir ou pour celui des spectateurs. Ils ne s'exercent pas en vue d'une action « utile » future.

\section{Plaisir et souffrance au travail}

On pourrait dire que ce qui définit le jeu, c'est qu'on agit pour le plaisir de la réussite, et que la réussite elle-même n'a pas d'autre utilité que le plaisir qu'elle produit. C'est même, me semble-t-il, une évidence, pour ne pas dire une banalité. Ce qui est moins évident mais sur quoi je voudrais insister, c'est qu'il y a toujours une dimension de jeu, c'est-à-dire de plaisir, dans l'action technique. Les sociologues et les psychologues du travail ont été beaucoup plus enclins à dénoncer les souffrances liées au travail qu'à en décrire les aspects positifs. C'est oublier que l'inaction forcée entraîne des souffrances encore pires, dont l'ennui (qui n'est pas un mal bénin) n'est que la manifestation la plus évidente, mais dont les conséquences les plus profondes sont 


\author{
* Sur le plaisir et la souffrance \\ au travail \\ La multiplication des suicides à France \\ Télécom depuis quelques mois \\ (ceci est écrit en novembre 2009) \\ a donné à la question, ancienne, \\ de la souffrance au travail, \\ une visibilité médiatique \\ tout à fait nouvelle; la bibliographie \\ sest accrue en conséquence. \\ II m'est tout à fait impossible d'en \\ donner ici une idée tant soit peu \\ générale. Je ne peux que proposer \\ deux impressions assez subjectives. \\ La première est qu'on s'est toujours \\ beaucoup plus intéressé à la \\ souffrance au travail qu'au plaisir. \\ La seconde est qu'on tend trop à \\ imputer aux conditions de travail \\ proprement dites des souffrances \\ qui sont en fait bien davantage le \\ résultat d'un refus, par l'entourage, de \\ partager l'expérience de la personne \\ concernée, c'est-à-dire de reconnaître \\ la valeur ou la signification de son \\ travail; ce qui peut aller jusqu’à \\ lui refuser la possibilité même de \\ travailler (placardisation). \\ Les deux auteurs que je choisirais de \\ citer sur ce thème sont: \\ Dejours Christophe et Yves Clot \\ (cf. bibliogrpahie).
}

peut-être la perte de sens et d'identité qui se produisent lorsqu'il y a véritablement exclusion (ce que j'ai appelé plus haut aliénation sociale). Les êtres humains, s'identifient par ce qu'ils font, car c'est ce qu'ils font qui les fait exister. Quelqu'un qui ne ferait rien (hypothèse absurde) ne pourrait pas exister, pas plus socialement que physiquement. Activité et identité sont inséparables. L'utilité (économique) a une importance déterminante, dans la mesure où, en dehors du jeu, il ne peut pas exister (autre hypothèse absurde) d'activités foncièrement inutiles. Mais sur le plan des motivations de l'action, l'utilité ne vient qu'en second. Ce qui vient en premier, c'est le désir d'identité, désir qui ne peut être satisfait que par la conscience d'appartenir à un groupe, non pas passivement comme un simple numéro, mais comme quelqu'un dont le savoir-faire est reconnu par les autres. Il y a dans cette reconnaissance un véritable plaisir, qui, c'est vrai, s'exprime assez rarement, mais qu'on peut mesurer aux souffrances qui surviennent quand cette reconnaissance est refusée, quand le lien est rompu ${ }^{3}$. Laction technique me socialise, m'identifie, fait de moi tel membre de tel groupe, c'est-à-dire une personne. C'est pourquoi j'y prends toujours du plaisir, même quand les tâches qui me sont affectées sont subalternes, pénibles, répétitives.

\section{Technique, économie et philosophie}

Je crois que la méconnaissance de ces aspects est l'une des faiblesses les plus sérieuses de la recherche en technologie. Cela est particulièrement visible chez les historiens qui, pour la plupart, ne voient les techniques que par le prisme de l'économie. Ils sont en cela, me semble-t-il, les héritiers d'une tradition ancienne, née en Angleterre au début du xix siècle, reprise ensuite par Marx et ses épigones. Encore une fois, je n'ai rien contre l'économie, dont je ne songe pas à contester l'importance. Mais il ne faut pas voir les choses que sous leur aspect économique. Pour les marxistes par exemple, les techniques font partie des «forces productives", ce qui est une vision proprement économiste. Étant donné que le « primat des forces productives » est si souvent cité dans leur phraséologie, on pouvait s'attendre à ce que les chercheurs marxistes fussent plus nombreux que les autres à s'intéresser aux techniques et qu'ils fissent vigoureusement progresser les connaissances dans ce domaine. Il n'en a rien été. Cette stérilité tient sans doute à des raisons multiples, mais il me semble que parmi ces raisons, l'économisme vient en premier. Dans la perspective économiste, la technique n'a d'intérêt que comme moyen, il n'y a donc pas de place pour des analyses plus détaillées que celles qui peuvent servir à illustrer telle ou telle théorie. La machine à vapeur est intéressante (je simplifie) dans la mesure où elle est censée avoir produit le capitalisme industriel. S'y intéresser pour d'autres raisons serait perdre son temps; ce genre de curiosités doit être laissé à ces maniaques inoffensifs que sont les collectionneurs. 
Les marxistes ne sont pas seuls en cause. Encore une fois, l'économisme est depuis fort longtemps l'idéologie dominante chez beaucoup d'historiens qui ne sont rien moins que marxistes, y compris et peut-être surtout dans les pays de langue anglaise. Je n'ose pas trop mavancer en ce qui concerne l'histoire industrielle. Mais pour l'histoire de l'agriculture, c'est flagrant. En Angleterre tout particulièrement, mais aussi en France, qui a largement suivi sur ce point le modèle anglais, la bibliographie d'histoire agraire accumulée depuis un siècle et demi est immense. On y trouve tout sur tous les aspects de la question, sauf sur l'agriculture elle-même en tant qu'activité technique, qui (malgré quelques belles exceptions) est à peu près totalement ignorée.

Je n'ai pas parlé des philosophes, de part et d'autre de la Manche ou du Rhin. Je voudrais en évoquer deux qui sont à mon avis d'une importance d'autant plus grande qu'elle est méconnue: Bergson et Simone Weil. Dans L'Évolution créatrice (1907), Bergson affirme que l'intelligence humaine s'est formée dans et pour la manipulation des corps solides, et qu'ainsi, l'homme aurait dû se nommer lui-même «Homo faber » plutôt qu'Homo sapiens. Dans ses Réflexions sur les causes de la liberté et de l'oppression sociale (écrites en 1934), Simone Weil fait un pas de plus. La matière est

"sans indulgence et sans perfidie», dit-elle; c'est pourquoi, dans l'action outillée, « la pensée doit se soustraire au désir et à la crainte, et s'appliquer uniquement à établir un rapport exact entre les mouvements imprimés aux instruments et le but poursuivi [...] L'attention se porte exclusivement sur les combinaisons formées par les mouvements de la matière inerte, et la notion de nécessité

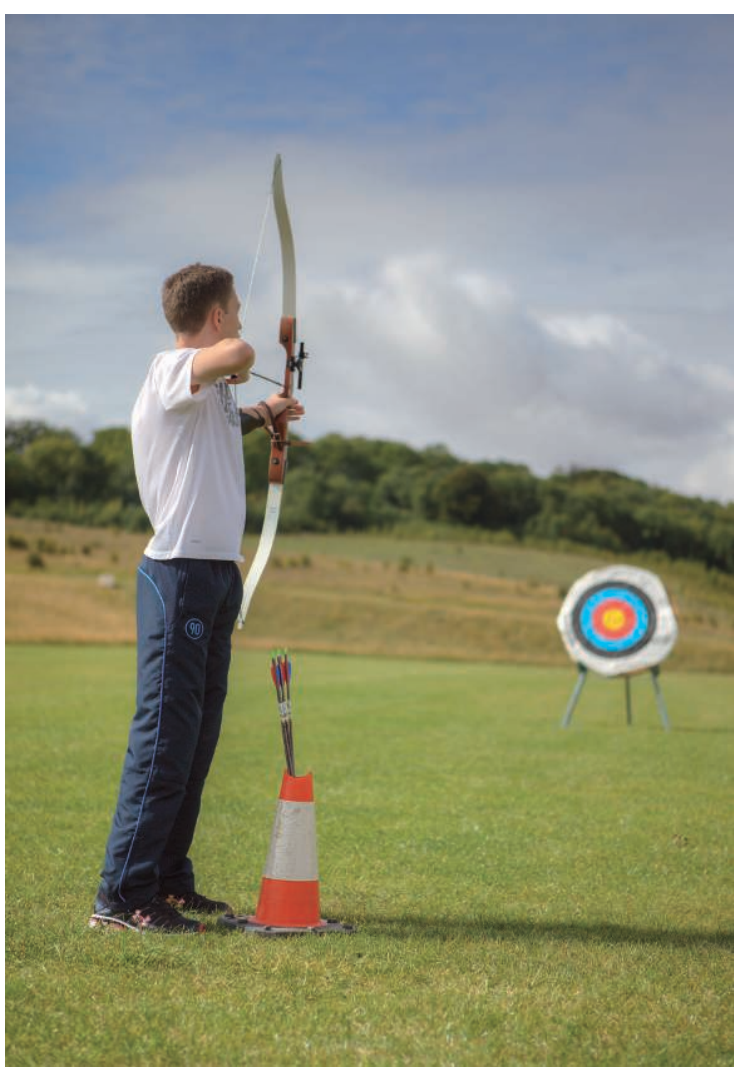

(1) Bill Hails

Pour elle, autrement dit, l'action technique est le domaine dans lequel l'homme fait l'apprentissage d'une réalité indépendante de ses désirs et de ses craintes. Apprentissage qui le conduira à la notion de nécessité, puis à la construction de la conscience d'autrui et de soi-même.

Je ne peux pas développer ces idées ici, bien que je les croie absolument fondamentales. Je le peux d'autant moins que ni Henri Bergson ni Simone Weil n'ont pu ou voulu les développer. Je voudrais seulement rappeler que l'œuvre de Bergson eut un succès extraordinaire, non seulement en France mais dans toute l'Europe, et que ce succès a engendré, du début au milieu $\mathrm{du} \mathrm{xx}^{\mathrm{e}}$ siècle, une prolifération d'écrits sur les techniques dont nous n'avons plus l'idée aujourd'hui. Il y a évidemment de tout dans cette masse d'écrits, que je n'ai moi-même qu'à peine explorés. Mais il faut savoir que c'est dans cette atmosphère que sont nés les auteurs de référence que sont devenus pour nous un Haudricourt ou un Leroi-Gourhan. Mauss lui-même doit à Bergson de s'être écarté après 1920 de la stricte orthodoxie durkheimienne qui excluait les techniques de la sociologie. 
La technologie a un passé plus riche que nous ne le pensons, et il y a de bonnes raisons de vouloir renouer avec ce passé. D'abord parce que c'est le seul moyen de nous garantir contre la répétition des mêmes erreurs. Mais surtout parce que c'est aussi un moyen de comprendre comment et pourquoi les attitudes des chercheurs actuels sont différentes en Allemagne, en Angleterre, en France, en Italie, au Japon, etc. Autant de pays, autant de traditions qui ont leurs forces et leurs faiblesses. Comparer ces traditions semble un bon moyen de mieux s'entendre.

\section{NOTES}

1. Pour les références exactes, cf. Sigaut 1998, note 8.

2. Cf. notamment Sigaut 1990, 1991, 1992, 1994, 2002 , 2004 et 2007.

3. Sigaut 1990, 1992.

4. D’où par exemple le véritable drame vécu par ce petit garçon qui veut montrer à son père qu'il va plonger, ce à quoi son père refuse violemment de s'intéresser. Voir le dessin de Riad Sattouf paru dans Charlie Hebdo n 728 du 31 mai 2006.

\section{RÉFÉRENCES}

Ampère, André-Marie, 1843-56, Essai sur la philosophie des sciences. Paris, Bachelier.

Bergson, Henri, 1907, L'Évolution créatrice, Bibliothèque des philosophies contemporaines. Paris, F. Alcan.

Clot Yves, 1999, La Fonction psychologique du travail. Paris, PUF.

Darré, Jean-Pierre, 1985, La Parole et la technique. Paris, L'Harmattan.

Dejours, Christophe, 1993, Travail : usure mentale - essai de psychopathologie du travail. Paris, Bayard.

Dejours, Christophe, 1998, Souffrance en France. Paris, Seuil (Coll. L'Histoire immédiate).

Guédez, Annie, 1994, Compagnonnage et apprentissage. Paris, PUF.

Sigaut, François,

-1987, « Renouer le fil », Techniques \& culture n 9 : 1-15.

-1987, « Des Idées pour observer », ibid. n $10: 1-12$.

-1990, « Folie, réel et technologie », ibid. n 15: 167-179 [rééd. dans Travailler, 12, 2004 : 117-134].

-1991, «L'Apprentissage vu par les ethnologues... », in D. Chevallier éd., Savoir faire et pouvoir transmettre,

Paris, Éditions de la Maison des sciences de l'homme : 33-42.

-1992, « Le Triangle du sens », Techniques \& culture n 19: 201-209.

-1994, « Technology », in T. Ingold (éd.), Companion Encyclopaedia of Anthropology, Londres et New York, Routledge : 420-459.

-1998, « Défense et illustration de la technographie », in R. Guesnerie \& F. Hartog éds., Des Sciences et des techniques: un débat, Éditions de l'EHESS (Cahier des Annales) : 289-301.

-2002, « La Formule de Mauss », ibid., 40: 153-168.

-2004, « Les Techniques dans la pensée narrative », ibid., 43-44: 191-214.

-2007, « Les Outils et le corps », Communications, 81: 9-30.

Weil, Simone, 1955, Réflexions sur les causes de la liberté et de l'oppression sociale. Paris, Galimard (Coll. L'Histoire immédiate). 


\section{RÉSUMÉ}

Techniques, technologies, apprentissage et plaisir au travail... Lauteur analyse les différentes traditions en anthropologie des techniques sous l'angle des difficultés sous-jacentes au sujet même de ces études. La première difficulté concerne la confusion entre technique et technologie, termes employés dans des acceptions différentes. La seconde concerne la question de l'expérience et des compétences techniques acquises par le chercheur, lequel doit à la fois apprendre à faire et apprendre à expliquer à d'autres comment on fait. La suivante touche à l'expérience technique commune, partagée par les différents membres d'un groupe social et qui s'ancre dans l'efficacité matérielle. La notion d'efficacité matérielle étant elle-même souvent confondue avec celle d'utilité, au sens économique étroit du terme. Cette dernière difficulté tient à un point de vue trop étroitement économique sur les techniques, y compris chez les marxistes, qui, bien que d'une importance réelle, a fréquemment restreint la technique au rôle de moyen. Enfin, l'auteur attire l'attention sur la richesse des études des techniques du début et du milieu du xx siècle, due à l'influence d'écrits philosophiques tels que ceux de Bergson et de Simone Weil, richesse à ce jour encore peu explorée. C'est, conclut-il, en prenant en compte ces recherches passées en comparant les différentes traditions auxquelles elles appartiennent, que l'on pourra surmonter ces différentes difficultés.

\section{ABSTRACT}

Techniques, technologies, training and pleasure in work... The author analyses the different traditions in the anthropology of techniques through a series of underlying difficulties, related to the subject itself. The first diffculty is with the confusion between techniques and technology, the definition of which varies. The second is with the question of a researcher's technical skills and expertise: the researcher must not only learn how to do, but also how to explain to others how to do. The next difficulty relates to the common technical experience, shared by different members of a social group, experience that is anchored within material efficacy. This notion of material efficacy is itself too often confused with the notion of usefulness, in its narrow economical sense. It is the same narrow economical understanding, including in Marxist approaches, which has often constrained techniques to a role of means. Finally, the author points out the often forgotten richness of studies on techniques of the beginning and the mid-20th century, influenced notably by philosophical works such as Bergson's or Simone Weil's. He concludes by arguing that the studies of these works and the comparisons of the different traditions to which they belong can help overcome the difficulties inherent to the anthropology of techniques.

\section{MOTS CLÉS}

Technique, technologie, efficacité, utilité, expérience partagée.

\section{KEYWORDS}

Technique, technology, efficacy, usefulness, shared experience. 